\title{
BONE
}

\section{Osteocalcin influences fetal brain development and adult brain function}

Few studies have investigated the effects of osteocalcin on the brain. In a recent paper, a team led by Gerard Karsenty (Columbia University, USA) has shown that osteocalcin crosses the placenta and the blood-brain barrier to influence fetal development and brain function after birth in mice.

"During development, osteocalcin from the mother crosses the placenta and goes into the brain of the fetus, where it is needed for the formation of the hippocampus and therefore the establishment of memory," says Karsenty. Maternal osteocalcin also prevented neuronal apoptosis in the embryo. Thus, the severity of neuroanatomical defects and learning difficulties in osteocalcin null mice was determined by the genotype of their mother. In addition, when pregnant osteocalcin null mice were given osteocalcin, their osteocalcin null offspring did not develop brain defects or learning difficulties.
In adult mice, the researchers found that osteocalcin was able to cross the bloodbrain barrier. Osteocalcin then bound to neurons of the brainstem, midbrain and hippocampus, which enabled it to enhance the synthesis of monoamine neurotransmitters and inhibit the synthesis of GABA, which led to reduced anxiety and depression and improved learning and memory.

The skeleton, via osteocalcin, regulates cognition and is a mediator of the maternal influence on the brain development and function of the offspring, note the authors. "We need to tease out the mode of action of osteocalcin in the brain and to identify all sites of action of osteocalcin in the brain," concludes Karsenty.

\section{Claire Greenhill}

Original article Oury, F. et al. Maternal and offspring pools of osteocalcin influence brain development and functions. Cell 155, 228-241 (2013) 